\title{
Joseph Geraci Responds
}

To the Editor:

Although I disagree with the parameters of research that Michael Schaefer places on the NDE, I truly appreciate his interest and com- 
ments. I believe that dialogue regarding the NDE is necessary if its meaning is to be understood and shared. Perhaps one day the concerns of Schaefer and other researchers will be answered when the NDEr is able to verbalize the essence of the experience, which yet remains locked inside.

Joseph B. Geraci 105 Ten Acre Road New Britain, CT 06052 